\title{
BRIGHT STAR CLUSTERS IN THE ANTENNAE ANALYZED WITH EVOLUTIONARY SYNTHESIS
}

\author{
UTA FRITZE - V. ALVENSLEBEN AND OLIVER KURTH \\ Universitäts-Sternwarte, Göttingen, Germany
}

\section{Introduction}

The Antennae galaxies (NGC 4038/39) are a pair of relatively nearby $\left(D \sim 19 \mathrm{Mpc}\right.$ for $\left.H_{0}=75\right)$ gas-rich spirals of comparable mass in the process of merging with extensive dynamical and evolutionary synthesis modeling available. With HST WFPC1 Whitmore \& Schweizer (1995) (WS95) detected 738 bright Young Star Clusters (YSCs) around the Antennae. YSCs have been detected in many interacting and starbursting galaxies, but the YSC system in the Antennae is the most populous one, well defining an exponential Luminosity Function (LF) over more than 5 mag above the completeness limit. Problems arising from crowding of the YSCs on a bright and variable galaxy background and from the PSF of WFPC1 may cause blending of YSCs and an overestimation of their effective radii.

A question with far-reaching consequences, e.g. for the formation of elliptical galaxies, is if the YSCs formed in this interaction-triggered starburst are open or (proto-) globular clusters (GCs).

Concentration parameters involving the tidal radius being inaccessible to observations as a distinction criterion, interest focuses on the LF. While in the Milky Way and nearby galaxies the LF is exponential for open clusters but Gaussian for old GC systems, the LF of a young GC system is unknown.

So, the question - if GCs are formed in mergers ? - gets related to the question as to the evolution of a GC system's LF. Vesperini (1997) shows that the strong dynamical effects on a GC system do not change the shape of its mass function, provided it starts from a Gaussian. The aim of this paper is to study the effects of the spectrophotometric evolution of the YSC system on its LF. 
The formation of $G C s$ is expected in gas-rich spiral-spiral mergers on the basis of the high star formation efficiencies in mergers and merger remnants (Fritze - v. Alvensleben \& Gerhard 1994a,b, Kurth 1996) together with hydrodynamical modeling that requires high star formation efficiences for GC formation (Brown et al. 1995)

\section{Evolution of Star Clusters and Metallicities}

The evolution of star clusters for 5 different metallicities $10^{-4} \leq \mathrm{Z} \leq 0.04$ is modeled as in Fritze - v. Alvensleben \& Burkert (1995, FB95). Magnitudes, colours, stellar metallicity indicators, and synthetic spectra are calculated as a function of time and metallicity. Cluster metallicities can be predicted on the basis of the spiral progenitors' ISM abundances (Fritze - v. Alvensleben \& Gerhard 1994a). Our prediction of $Z \sim 0.01$ for NGC 7252 , an Sc-Sc merger like the Antennae, is confirmed by spectroscopy of the brightest cluster W3 in NGC 7252 by Schweizer \& Seitzer (1993). For lack of spectroscopy and by analogy, we assume $Z \sim 0.01$ also for the YSCs in the Antennae. Once cluster metallicities are known precise age dating from $V-I$ or $U-V$ colours becomes possible.

Alone from their mean age of $\sim 1.3$ Gyr most of the YSCs in NGC 7252 are expected to be young GCs (FB95).

\section{Age Distribution of YSCs in the Antennae}

The mean V - I colour together with an average internal reddening correction results in a mean age of the YSC population in the Antennae of $0.2 \pm 0.2 \mathrm{Gyr}$, in agreement with global starburst age and dynamical time since pericenter (Kurth 1996, Barnes 1988).

Assuming a uniform age for the YSCs the time evolution of the LF simply is a shape-conserving shift towards fainter magnitudes. For a subsample of YSCs with effective radii $R_{\text {eff }} \leq 10 \mathrm{pc}$ Fritze $-\mathrm{v}$. Alvensleben (1996) shows that their LF after $\sim 12$ Gyr of evolution becomes compatible with a "normal" GC LF at least up to the turnover, though with some overpopulation of the faintest bins. Since these are most susceptible to depopulation by dynamical destruction and since the colour distribution also agrees with its Milky Way counterpart she argues that - at least for YSCs with $R_{\text {eff }} \leq 10 \mathrm{pc}$ the LF does not preclude them from being GCs (at variance with van den Bergh's (1995) statement for the entire YSC sample).

In an ongoing starburst like in the Antennae, the age spread among star clusters may be comparable to their ages. Therefore we analyze the age distribution of the YSCs as derived from their individual observed colours in $V-I$ and, as far as available, $U-V$. Fig. 1 clearly reveals two distinct age populations: a large population of 399 (probably 481) YSCs 


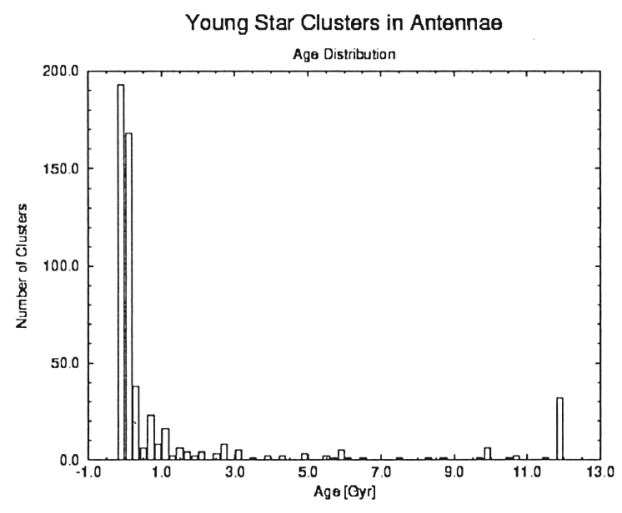

Figure 1. Age distribution of bright star clusters in the Antennae.

with ages from $0-2 \cdot 10^{8} \mathrm{yr}$ from the present burst and a small population of 32 (probably 69) old GCs from the parent galaxies. The small number of interlopers is probably due to inhomogeneous internal reddening (see Fritze - v. Alvensleben 1998). Strikingly, the number of old GCs agrees with the number expected in case the Milky Way and M31 were merging instead of NGC 4038 and 4039.

The average as well as the distribution of effective radii is not significantly different for the old GC and the YSC subsamples. Statistical tests assure the fraction of blended pairs to be $<10 \%$ even among clusters with $R_{\text {eff }}>10$ pc (see Fritze - v. Alvensleben 1998 for details).

\section{Evolution of the LF of the YSCs in the Antennae}

Once we have individual ages of the YSCs our models also allow to calculate their individual fading until a common age of say 12 Gyr. Meurer (1995) already suspects that age spread effects might change the shape of the LF in the course of evolution. The brightest clusters tend to be the youngest and will therefore fade more, while part of the faintest clusters will fade less than average. We find that not only the LF of the subsample of YSCs with $R_{\text {eff }} \leq 10$ pc evolves into a "perfectly normal" Gaussian, but also the $\mathrm{LF}$ of the entire YSC sample (see Fig. 2). The turnover occurs at $M_{\mathrm{V}_{\mathrm{o}}} \sim$ $-6.9 \mathrm{mag}$, more than $1 \mathrm{mag}$ brighter than the (evolved) completeness limit. The difference of $\sim 0.2 \mathrm{mag}$ to the typical elliptical galaxy GC systems' $M_{\mathrm{V}_{\mathrm{o}}} \sim-7.1 \mathrm{mag}$ (Harris 1991, Ashman et al. 1995) is a consequence of the enhanced metallicity of this secondary cluster population.

We conclude: Properly accounting for age spread effects and the resulting differences in fading the observed exponential LF of the entire YSC system around the merging Antennae galaxies evolves into a "typical" Gaussian GC LF over 12 Gyr (cf. Fritze - v. Alvensleben 1998). 


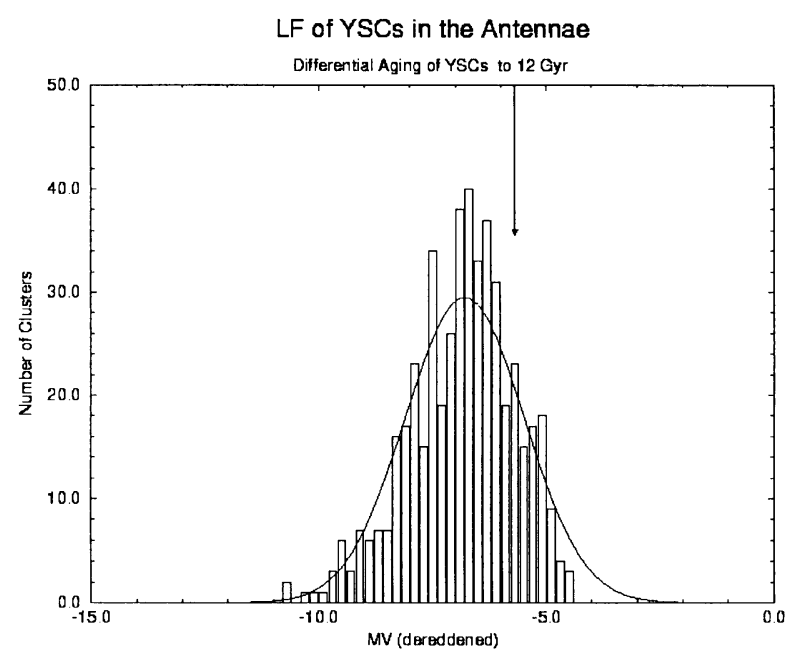

Figure 2. LF of YSCs in the Antennae as calculated from individual ages together with the resulting individual fading until 12 Gyr for every cluster. A Gaussian with $\left\langle\mathrm{M}_{\mathrm{V}_{0}}\right\rangle=-6.9 \mathrm{mag}$ and $\sigma\left(\mathrm{M}_{\left.\mathrm{V}_{0}\right)}\right)=1.3 \mathrm{mag}$ is overplotted, normalized to the number of clusters in the histogram. The vertical arrow marks the evolved observational completeness limit.

Together with Vesperini's (1997) result that dynamical effects destroying as much as $60 \%$ of an original GC population do not change its mass function if this initially had a Gaussian shape our results indicate that while there surely will be some open clusters among the YSC population - the bulk of the YSCs in the Antennae may well be young GCs. Their number is large enough for the Antennae to transform into an elliptical with "normal" specific GC frequency.

UFvA greatfully acknowledges a travel grant (Fr 916/4-1) from the DFG.

\section{References}

Ashman, K. M., Conti, A., Zepf, S. E., 1995, AJ 110, 1164

Barnes, J. E., 1988, ApJ 331, 699

Brown, J. H., Burkert, A., Truran, J. W., 1995, ApJ 440, 666

Fritze - v. Alvensleben, U.,1998, A\&A submitted

Fritze - v. Alvensleben, U., Burkert, A., 1995, A\&A 300, 58, (FB95)

Fritze - v. Alvensleben, U., Gerhard, O.E., 1994a, A\&A 285, 751

Fritze - v. Alvensleben, U., Gerhard, O.E., 1994b, A\&A 285, 775

Harris, W. E., 1991, ARAA 29, 543

Kurth, O., 1996, Diploma Thesis, Univ. Göttingen

Meurer, G. R., 1995, Nature 375, 742

Schweizer, F., Seitzer, P., 1993, ApJ 417, L29

van den Bergh, S., 1995, Nature 374, 215

Vesperini, E., 1997, MNRAS 287, 915

Whitmore, B. C., Schweizer, F., 1995, ApJ 420, 87

Zepf, S. E., Ashman, K. M., 1993, MNRAS 264, 611 